\title{
On the Reynolds-averaged Navier-Stokes Equations
}

\author{
Bohua $\operatorname{Sun}^{1}$ \\ 1 School of Civil Engineering 83 Institute of Mechanics and Technology, \\ Xi'an University of Architecture and Technology, Xi'an 710055, China \\ sunbohua@xauat.edu.cn
}

\begin{abstract}
This paper attempts to clarify an issue about the number of unknowns in the Reynolds-Averaged Navier-Stokes equations (RANS). The study discovers that all perspectives regarding the numbers of unknowns in the RANS stems from the misinterpretation of the Reynolds stress tensor. The current literature considers that the Reynolds stress tensor has six unknowns; however, this paper shows that the Reynolds stress tensor actually has only three unknowns, namely the three components of fluctuation velocity. The transport equation on both the Reynolds stress tensor and kinetic energy are discussed as well. As application, the turbulent Berger's equation and Prandtl boundary layer equations are resolved. All solutions have no any adjustable parameters. The paper also propose a general algorithm for three dimensional turbulence flow. It is found that the magnitude of velocity fluctuations or turbulence are proportional to the flow pressure, which is a remarkable discovery.
\end{abstract}

Keywords: turbulence; the Reynolds stress tensor; turbulence closure problem

\section{INTRODUCTION}

Turbulence is everywhere, controlling the drag on cars, airplanes, and trains, whilst dictating the weather through its influence on large-scale atmospheric and oceanic flows. Even solar flares are a manifestation of turbulence since they are triggered by vigorous motions on the surface of the Sun [1-40].

The study of turbulence is a difficult subject owing to its complex and forbidding mathematical descriptions, which is often considered as the last unsolved problem in classical physics. In 1895 Reynolds published a seminal work on turbulence [1], in which he proposed that flow velocity $\boldsymbol{u}$ and pressure $p$ are decomposed into its timeaveraged quantities, $\overline{\boldsymbol{u}}, \bar{p}$, and fluctuating quantities, $\tilde{\boldsymbol{u}}$, $\tilde{p}$; thus, the Reynolds decompositions are:

$$
\begin{aligned}
\boldsymbol{u} & =\overline{\boldsymbol{u}}(\boldsymbol{x})+\tilde{\boldsymbol{u}}(\boldsymbol{x}, t), \\
p(\boldsymbol{x}, t) & =\bar{p}(\boldsymbol{x})+\tilde{p}(\boldsymbol{x}, t),
\end{aligned}
$$

where coordinates and time are $(\boldsymbol{x}, t)$. According to Reynolds, $\overline{\boldsymbol{u}}$ represent a mean-motion at each point and $\tilde{\boldsymbol{u}}$ a motion at the same point relative to the mean-motion at the point. Therefore, the Reynolds called the $\overline{\boldsymbol{u}}$ mean-motion and $\tilde{\boldsymbol{u}}$ relative-mean-motion [1]. The time-averaging definition is given by $\overline{\boldsymbol{u}}(\boldsymbol{x})=$ $\lim _{T \rightarrow \infty} \frac{1}{T} \int_{t_{0}}^{t_{0}+T} \boldsymbol{u}(\boldsymbol{x}, t) d t$, and time-fluctuation $\tilde{\boldsymbol{u}}=\boldsymbol{u}-$ $\bar{u}(\boldsymbol{x})$.

\section{REYNOLDS-AVERAGED NAVIER-STOKES EQUATIONS}

With the decomposition the Navier-Stokes equation is then transformed into the Reynolds-averaged
Navier - Stokes equations (RANS) as follows

$$
\begin{aligned}
\rho \boldsymbol{\nabla} \cdot(\overline{\boldsymbol{u}} \otimes \overline{\boldsymbol{u}})+\nabla \bar{p} & =\mu \nabla^{2} \overline{\boldsymbol{u}}+\nabla \cdot \boldsymbol{\tau}, \\
\nabla \cdot \overline{\boldsymbol{u}} & =0,
\end{aligned}
$$

where dynamic viscosity $\mu$, gradient operator $\nabla=\boldsymbol{e}_{i} \nabla_{i}$, base vector in the i-coordinate $\boldsymbol{e}_{i}$, and tensor product $\otimes$, and the Reynolds stress tensor is given by

$$
\boldsymbol{\tau}=-\rho \overline{\tilde{\boldsymbol{u}} \otimes \tilde{\boldsymbol{u}}}=-\rho \lim _{T \rightarrow \infty} \frac{1}{T} \int_{t_{0}}^{t_{0}+T}(\tilde{\boldsymbol{u}} \otimes \tilde{\boldsymbol{u}}) d t
$$

where $T$ is the period of time over which the averaging takes place and must be sufficiently large to give meaningful averages and is needed to measure mean values depends on the accuracy desired. Reynolds stress is apparent stress owing to the fluctuating velocity field $\tilde{\boldsymbol{u}}$.

Denoting kinematic viscosity $\nu=\mu / \rho$, the above equations can be equivalently rewritten in conventional form:

$$
\begin{gathered}
\overline{\boldsymbol{u}} \cdot \boldsymbol{\nabla} \overline{\boldsymbol{u}}+\frac{1}{\rho} \boldsymbol{\nabla} \bar{p}+ \\
\lim _{T \rightarrow \infty} \frac{1}{T} \int_{t}^{t+T}(\tilde{\boldsymbol{u}} \cdot \boldsymbol{\nabla} \tilde{\boldsymbol{u}}) d t \\
=\nu \boldsymbol{\nabla}^{2} \overline{\boldsymbol{u}} \\
\boldsymbol{\nabla} \cdot \overline{\boldsymbol{u}}=0
\end{gathered}
$$

\section{HOW MANY UNKNOWNS ARE THERE IN THE RANS ?}

All current literatures and textbooks believe that the Reynolds stresses $\boldsymbol{\tau}$ has six unknowns (In the later, we will show that the Reynolds stresses have only three unknowns instead of six ones.). This understanding result$\mathrm{s}$ a consensus of saying that there are 10 unknowns in Eqs.3, 4 and/or Eqs.35, 35 .

If you carefully read Reynolds' paper in 1895 [1], and will see that Reynolds did not discussed the number of unknowns in the RANS. However, from his presentation we are sure that he never considered the term $\rho \overline{\tilde{\boldsymbol{u}} \otimes \tilde{\boldsymbol{u}}}$ as 
independent unknowns, while for some cases he even proposed explicit expressions for the velocity fluctuation $\tilde{\boldsymbol{u}}$, for instance, expressions in page 149 and Eq.(50) in page 158 of Reynolds paper [1], which means that he actually take the velocity fluctuation $\tilde{\boldsymbol{u}}$ as unknowns. Due to the limited references, the author has no idea who was the first person proposed this 10-unknowns perception on the RANS.

Clearly, the RANS in Eqs.3, 4 has four independent equations governing the mean velocity field; namely the three components of the Reynolds equation Eqs.3 together with the mean continuity equation Eq.4. However, these four equations contain more than four unknowns. In addition to $\overline{\boldsymbol{u}}$ and $\bar{p}$, there are also the Reynolds stresses $\boldsymbol{\tau}$, which results the Reynolds-averaged Navier-Stokes equations unclosed. The closure problem is being considered as the number one topic in turbulence, during the past several decades, scientists and engineers have made many attempts towards solving the closure problem.

Regarding the RANS closure problem, the curren$\mathrm{t}$ consensus is that there are six unknown components in the symmetric Reynolds stress tensor $\boldsymbol{\tau}$, namely $\tau_{11}, \tau_{12}, \tau_{13}, \tau_{22}, \tau_{23}, \tau_{33}$. However, we have a completely different perspectives. What we believe is that the Reynolds stress tensor $\boldsymbol{\tau}$ has only three unknowns, namely the velocity fluctuation components $u_{i}^{\prime}(i=1,2,3)$ due to the fact that the Reynolds stress tensor is simply an integration of a second order dyadic tensor of flow velocity fluctuations rather than a general symmetric tensor.

\section{LEMMAS}

To support our statement, let's us introduce some lemmas as follows

Lemma 1 Giving two vectors, $\boldsymbol{v}(\boldsymbol{x}, t)=v_{i} \boldsymbol{e}_{i}=v_{1} \boldsymbol{e}_{1}+$ $v_{2} \boldsymbol{e}_{2}+v_{3} \boldsymbol{e}_{3}$ and $\boldsymbol{w}(\boldsymbol{x}, t)=w_{j} \boldsymbol{e}_{j}=w_{1} \boldsymbol{e}_{1}+w_{2} \boldsymbol{e}_{2}+w_{3} \boldsymbol{e}_{3}$, we can defined a dyadic tensor $\boldsymbol{v} \otimes \boldsymbol{w}$ as follows [41]

$$
\begin{aligned}
\boldsymbol{v} \otimes \boldsymbol{w} & =v_{i} \boldsymbol{e}_{i} \otimes w_{j} \boldsymbol{e}_{j}=v_{i} w_{j} \boldsymbol{e}_{i} \otimes \boldsymbol{e}_{j} \\
& =v_{1} w_{1} \boldsymbol{e}_{1} \otimes \boldsymbol{e}_{1}+v_{1} w_{2} \boldsymbol{e}_{1} \otimes \boldsymbol{e}_{2}+v_{1} w_{3} \boldsymbol{e}_{1} \otimes \boldsymbol{e}_{3} \\
& +v_{2} w_{1} \boldsymbol{e}_{2} \otimes \boldsymbol{e}_{1}+v_{2} w_{2} \boldsymbol{e}_{2} \otimes \boldsymbol{e}_{2}+v_{2} w_{3} \boldsymbol{e}_{2} \otimes \boldsymbol{e}_{3} \\
& +v_{3} w_{1} \boldsymbol{e}_{3} \otimes \boldsymbol{e}_{1}+v_{3} w_{2} \boldsymbol{e}_{3} \otimes \boldsymbol{e}_{2}+v_{3} w_{3} \boldsymbol{e}_{3} \otimes \boldsymbol{e}_{3}
\end{aligned}
$$

In general case of $\boldsymbol{v} \neq \boldsymbol{w}$, the dyadic tensor $\boldsymbol{v} \otimes \boldsymbol{w}$ is a general tensor with 9 components (elements), and have 6 unknowns, namely, $v_{1}, v_{2}, v_{3}$ and $w_{1}, w_{2}, w_{3}$, since the 9 components can be fully determined by the 6 unknowns.

If $\boldsymbol{v}=\boldsymbol{w}$, the tensor $\boldsymbol{v} \otimes \boldsymbol{w}$ is a 2rd order tensor with 9 components (elements), and have 3 unknowns, namely, $v_{1}, v_{2}, v_{3}$ and/or $w_{1}, w_{2}, w_{3}$, since the 9 components can be fully determined by the 3 unknowns.

Lemma 2 Giving three vectors, $\boldsymbol{u}(\boldsymbol{x}, t)=u_{i} \boldsymbol{e}_{i}=u_{1} \boldsymbol{e}_{1}+$ $u_{2} \boldsymbol{e}_{2}+u_{3} \boldsymbol{e}_{3}, \boldsymbol{v}(\boldsymbol{x}, t)=v_{i} \boldsymbol{e}_{i}=v_{1} \boldsymbol{e}_{1}+v_{2} \boldsymbol{e}_{2}+v_{3} \boldsymbol{e}_{3}$ and $\boldsymbol{w}(\boldsymbol{x}, t)=w_{j} \boldsymbol{e}_{j}=w_{1} \boldsymbol{e}_{1}+w_{2} \boldsymbol{e}_{2}+w_{3} \boldsymbol{e}_{3}$, we can defined $a$ 3rd order tensor $\boldsymbol{v} \otimes \boldsymbol{w}$ as follows

$\boldsymbol{u} \otimes \boldsymbol{v} \otimes \boldsymbol{w}=u_{i} \boldsymbol{e}_{k} \otimes v_{k} \boldsymbol{e}_{i} \otimes w_{j} \boldsymbol{e}_{j}=u_{k} v_{i} w_{j} \boldsymbol{e}_{k} \otimes \boldsymbol{e}_{i} \otimes \boldsymbol{e}_{j}$

In general case of $\boldsymbol{u} \neq \boldsymbol{v} \neq \boldsymbol{w}$, the tensor $\boldsymbol{u} \otimes \boldsymbol{v} \otimes \boldsymbol{w}$ is a general tensor with 27 components (elements), and have 9 unknowns, namely, $u_{1}, u_{2}, u_{3}, v_{1}, v_{2}, v_{3}$ and $w_{1}, w_{2}, w_{3}$, since the 27 components can be fully determined by the 9 unknowns.

If $\boldsymbol{u}=\boldsymbol{v}=\boldsymbol{w}$, the tensor $\boldsymbol{u} \otimes \boldsymbol{v} \otimes \boldsymbol{w}$ is a 3rd order tensor with 27 components (elements), and have 3 unknowns, namely, $u_{1}, u_{2}, u_{3}$, or $v_{1}, v_{2}, v_{3}$ and/or $w_{1}, w_{2}, w_{3}, s$ ince the 27 components can be fully determined by the 3 unknowns.

Lemma 3 Giving a vector, $\boldsymbol{v}(\boldsymbol{x}, t)=v_{i} \boldsymbol{e}_{i}=v_{1} \boldsymbol{e}_{1}+$ $v_{2} \boldsymbol{e}_{2}+v_{3} \boldsymbol{e}_{3}$, we can defined a 2nd order symmetric dyadic tensor $\boldsymbol{v} \otimes \boldsymbol{v}$ and its mean value $\boldsymbol{A}(\boldsymbol{x})$ as follows

$$
\begin{aligned}
\boldsymbol{A}(\boldsymbol{x}) & =\lim _{T \rightarrow \infty} \frac{1}{T} \int_{t_{0}}^{t_{0}+T} \boldsymbol{v} \otimes \boldsymbol{v} d t \\
& =\lim _{T \rightarrow \infty} \frac{1}{T} \int_{t_{0}}^{t_{0}+T} v_{i} v_{j} \boldsymbol{e}_{i} \otimes \boldsymbol{e}_{j} d t \\
& =\lim _{T \rightarrow \infty} \frac{1}{T} \int_{t_{0}}^{t_{0}+T}\left[v_{1} v_{1} \boldsymbol{e}_{1} \otimes \boldsymbol{e}_{1}+v_{1} v_{2} \boldsymbol{e}_{1} \otimes \boldsymbol{e}_{2}\right. \\
& +v_{1} v_{3} \boldsymbol{e}_{1} \otimes \boldsymbol{e}_{3}+v_{2} v_{1} \boldsymbol{e}_{2} \otimes \boldsymbol{e}_{1} \\
& +v_{2} v_{2} \boldsymbol{e}_{2} \otimes \boldsymbol{e}_{2}+v_{2} v_{3} \boldsymbol{e}_{2} \otimes \boldsymbol{e}_{3} \\
& +v_{3} v_{1} \boldsymbol{e}_{3} \otimes \boldsymbol{e}_{1}+v_{3} v_{2} \boldsymbol{e}_{3} \otimes \boldsymbol{e}_{2} \\
& \left.+v_{3} v_{3} \boldsymbol{e}_{3} \otimes \boldsymbol{e}_{3}\right] d t
\end{aligned}
$$

where $v_{i} v_{j}=v_{j} v_{i}$. Although $\boldsymbol{A}(\boldsymbol{x})$ has six independen$t$ components, namely $v_{1} v_{1}, v_{1} v_{2}, v_{1} v_{3}, v_{2} v_{2}, v_{2} v_{3}, v_{3} v_{3}$, however it is clear there are only three independent quantities, namely, $v_{1}, v_{2}, v_{3}$, in the $\boldsymbol{A}(\boldsymbol{x})$. It is because that the quantities $v_{1} v_{1}, v_{1} v_{2}, v_{1} v_{3}, v_{2} v_{2}, v_{2} v_{3}, v_{3} v_{3}$ can be fully determined by $v_{1}, v_{2}, v_{3}$.

The lemma 3 actually states that any (time) averaging operation is just a method of data processing and will not change the number of unknowns of the problem.

It is clear that $A(\boldsymbol{x})$ will be the Reynolds stress tensor $\boldsymbol{\tau}$, if replacing $v_{1}, v_{2}, v_{3}$ by the components of velocity fluctuations $\tilde{u}_{i}(i=1,2,3)$, respectively, namely, $v_{1}=$ $\tilde{u}_{1}, v_{2}=\tilde{u}_{2}, v_{3}=\tilde{u}_{3}$.

\section{PROOF 1}

From the Lemma, we can see that the Reynolds stress tensor has only three unknown components. This can be proved easily as follows: the Reynolds stress tensor can 
be defined by

$$
\begin{aligned}
\boldsymbol{\tau} & =-\rho \overline{\tilde{\boldsymbol{u}} \otimes \tilde{\boldsymbol{u}}} \\
& =-\rho \overline{\tilde{u}_{i} \boldsymbol{e}_{i} \otimes \tilde{u}_{j} \boldsymbol{e}_{j}}=-\rho \overline{\tilde{u}_{i} \tilde{u}_{j}} \boldsymbol{e}_{i} \otimes \boldsymbol{e}_{j} \\
& =-\rho \lim _{T \rightarrow \infty} \frac{1}{T} \int_{t_{0}}^{t_{0}+T}\left(\tilde{u}_{i} \tilde{u}_{j} \boldsymbol{e}_{i} \otimes \boldsymbol{e}_{j}\right) d t \\
& =\left[-\rho \lim _{T \rightarrow \infty} \frac{1}{T} \int_{t_{0}}^{t_{0}+T} \tilde{u}_{i} \tilde{u}_{j} d t\right] \boldsymbol{e}_{i} \otimes \boldsymbol{e}_{j} \\
& =\tau_{i j} e_{i} \otimes \boldsymbol{e}_{j},
\end{aligned}
$$

and the fluctuation velocity convective terms are given by:

$$
\begin{aligned}
& \overline{\tilde{\boldsymbol{u}} \cdot \boldsymbol{\nabla} \tilde{\boldsymbol{u}}}=\overline{\tilde{u}_{i} \boldsymbol{e}_{i} \cdot\left[\boldsymbol{e}_{k} \partial_{k} \otimes\left(\tilde{u}_{j} \boldsymbol{e}_{j}\right)\right]} \\
& =\overline{\tilde{u}_{i} \tilde{u}_{j, k}} \boldsymbol{e}_{i} \cdot\left(\boldsymbol{e}_{k} \otimes \boldsymbol{e}_{j}\right)=\overline{\tilde{u}_{i} \tilde{u}_{j, k}}\left(\boldsymbol{e}_{i} \cdot \boldsymbol{e}_{k}\right) \boldsymbol{e}_{j} \\
& =\overline{\tilde{u}_{i} \tilde{u}_{j, k}} \delta_{i k} \boldsymbol{e}_{j}=\overline{\tilde{u}_{i} \tilde{u}_{j, i}} \boldsymbol{e}_{j} \\
& =\left(\lim _{T \rightarrow \infty} \frac{1}{T} \int_{t}^{t+T} \tilde{u}_{i} \tilde{u}_{j, i} d t\right) \boldsymbol{e}_{j} \\
& =\tau_{i j, i} \boldsymbol{e}_{j},
\end{aligned}
$$

where the Reynolds stress tensor in index format $\tau_{i j}$ is defined by

$$
\tau_{i j}=\tau_{j i}=-\rho \lim _{T \rightarrow \infty} \frac{1}{T} \int_{t_{0}}^{t_{0}+T} \tilde{u}_{i} \tilde{u}_{j} d t .
$$

It is clear that any $\tau_{i j}$ can be calculated by fluctuation velocity components $\tilde{u}_{1}, \tilde{u}_{2}, \tilde{u}_{3}$, which means that the $\tau_{i j}$ are dependent on the components $\tilde{u}_{1}, \tilde{u}_{2}, \tilde{u}_{3}$. In other words, the components $\tilde{u}_{1}, \tilde{u}_{2}, \tilde{u}_{3}$ are real unknowns. It is the key point why we say that the Reynolds stress tensor $\boldsymbol{\tau}$ has 6 unknowns is a big mistake in the turbulence modelling.

Therefore, the formulation in Eq.11 reveals that the Reynolds stress tensor $\boldsymbol{\tau}=-\rho \overline{\tilde{\boldsymbol{u}} \otimes \tilde{\boldsymbol{u}}}$ can be fully calculated by three independent components of fluctuation velocity $\tilde{u}_{1}, \tilde{u}_{2}, \tilde{u}_{3}$. In other words, the Reynolds stress tensor has only three unknowns rather than six ones. It means that the averaging technique is just a mathematical processing, which can provide us a mean value, however, can't change the number of unknowns of the problem.

The misinterpretation regarding the numbers of unknown components of all literature may stem from considering the Reynolds stress tensor as a general 2nd order symmetric tensor with six independent components. However, the Reynolds stress tensor is not an arbitrary 2nd order tensor, actually each of its component is made by the bi-product of fluctuation velocity components, which means that the Reynolds stress tensor is an dyadic tensor of the velocity fluctuation. The unknown components to construct the dyadic tensor are the three components of fluctuation velocity $\tilde{\boldsymbol{u}}$. Therefore, the Reynolds stress tensor has only three unknowns, namely $\tilde{u}_{1}, \tilde{u}_{2}, \tilde{u}_{3}$. For two dimensional flow, of course, the 2D Reynolds stress tensor has only two unknowns, namely $\tilde{u}_{1}, \tilde{u}_{2}$.

\section{PROOF 2}

Given two scalar functions, $\tilde{u}=\sqrt{2} U \cos (\omega t)$ and $\tilde{v}=$ $\sqrt{2} V \cos (\omega t+\theta)$, as independent unknowns, we can use them to construct a 2 nd order tensor or matrix with 4 components as follows: $(\tilde{u})^{2}, \tilde{u} \tilde{v}, \tilde{v} \tilde{u},(\tilde{v})^{2}$.

Thus time averages give:

$$
\begin{aligned}
\lim _{T \rightarrow \infty} \frac{1}{T} \int_{t_{0}}^{t_{0}+T}(\tilde{u} \tilde{u}) d t & =U^{2} \\
\lim _{T \rightarrow \infty} \frac{1}{T} \int_{t_{0}}^{t_{0}+T}(\tilde{u} \tilde{v}) d t & =U V \cos \theta \\
\lim _{T \rightarrow \infty} \frac{1}{T} \int_{t_{0}}^{t_{0}+T}(\tilde{v} \tilde{u}) d & =U V \cos \theta \\
\lim _{T \rightarrow \infty} \frac{1}{T} \int_{t_{0}}^{t_{0}+T}(\tilde{v} \tilde{v}) d t & =V^{2}
\end{aligned}
$$

In the definition of the Reynolds stress tensor, $\theta=0$, we have $\tilde{u}=\sqrt{2} U \cos (\omega t)$ and $\tilde{v}=\sqrt{2} V \cos (\omega t)$ and their averaging

$$
\begin{aligned}
& \lim _{T \rightarrow \infty} \frac{1}{T} \int_{t_{0}}^{t_{0}+T}(\tilde{u} \tilde{u}) d t=U^{2} \\
& \lim _{T \rightarrow \infty} \frac{1}{T} \int_{t_{0}}^{t_{0}+T}(\tilde{u} \tilde{v}) d t=U V \\
& \lim _{T \rightarrow \infty} \frac{1}{T} \int_{t_{0}}^{t_{0}+T}(\tilde{v} \tilde{u}) d t=U V \\
& \lim _{T \rightarrow \infty} \frac{1}{T} \int_{t_{0}}^{t_{0}+T}(\tilde{v} \tilde{v}) d t=V^{2}
\end{aligned}
$$

Therefore, the Reynolds stress tensor is given by

$$
\begin{aligned}
\boldsymbol{\tau} & =-\rho\left(U^{2} \boldsymbol{e}_{1} \otimes \boldsymbol{e}_{1}+U V \boldsymbol{e}_{1} \otimes \boldsymbol{e}_{2}\right. \\
& \left.+V U \boldsymbol{e}_{2} \otimes \boldsymbol{e}_{1}+V^{2} \boldsymbol{e}_{2} \otimes \boldsymbol{e}_{2}\right)
\end{aligned}
$$

The above process shows that, $\tilde{u}$ or $U$, and $\tilde{v}$ or $V$, are the independent unknowns, with them we can get the Reynolds stress tensor $\boldsymbol{\tau}$.

If keeping the $\theta$, namely, then we are talking about the time correlation (autocorrelations) at the same point. The correlation between the same (Greek autos = self or same) fluctuating quantity measured at two different times (at the same point in space) is not itself very relevant to the behaviour of turbulence and its measurement requires a time delay mechanism (usually a tape recorder with movable heads or a digital sample-and-delay system).

In the same way, you can define the space correlation, however, all literatures and textbooks said that the 
Reynolds stress tensor is the one-point or single-point velocity fluctuation correlation. Therefore, $\theta=0$.

Even not setting $\theta=0$, the Reynolds stress still has three independent unknowns, namely the three components of velocity fluctuations. This is the key point of this article. The conclusion of saying that the RANS has only 7 independent unknowns is still correct.

\section{PROOF 3}

To support this obvious statement from other point of view, let's study the problem by tensor representation theory and write the Reynolds stress tensor as follows:

$$
\boldsymbol{\tau}=-\rho \lim _{T \rightarrow \infty} \frac{1}{T} \int_{t_{0}}^{t_{0}+T} \boldsymbol{\sigma} d t
$$

where $\boldsymbol{\sigma}=\tilde{\boldsymbol{u}}(\boldsymbol{x}, t) \otimes \tilde{\boldsymbol{u}}(\boldsymbol{x}, t)=\tilde{u}_{i} \boldsymbol{e}_{i} \otimes \tilde{u}_{j} \boldsymbol{e}_{j}=\tilde{u}_{i} \tilde{u}_{j} \boldsymbol{e}_{i} \otimes \boldsymbol{e}_{j}$.

The characteristic equation of $\boldsymbol{\sigma}$ is given by

$$
\lambda^{3}-I_{1} \lambda^{2}+I_{2} \lambda-I_{3}=0 .
$$

Note $\operatorname{tr}(\boldsymbol{\sigma})^{2}=\left(\tilde{u}_{1}\right)^{2}+\left(\tilde{u}_{2}\right)^{2}+\left(\tilde{u}_{3}\right)^{2}$ and $\operatorname{tr}(\boldsymbol{\sigma})^{2}=\operatorname{tr}\left(\boldsymbol{\sigma}^{2}\right)$, thus

$$
\begin{aligned}
I_{1} & =\operatorname{tr}(\boldsymbol{\sigma})=\left(\tilde{u}_{1}\right)^{2}+\left(\tilde{u}_{2}\right)^{2}+\left(\tilde{u}_{3}\right)^{2} \\
I_{2} & =I_{2}=\frac{1}{2}\left[\left(\operatorname{tr}(\boldsymbol{\sigma})^{2}-\operatorname{tr}\left(\boldsymbol{\sigma}^{2}\right)\right]=0\right. \\
I_{3} & =\operatorname{det}(\boldsymbol{\sigma})=\varepsilon_{i j k}\left(u_{1} u_{j}\right)\left(u_{2} u_{j}\right)\left(u_{3} u_{j}\right) \\
& =\left(\tilde{u}_{1} \tilde{u}_{2} \tilde{u}_{3}\right)^{2}-\left(\tilde{u}_{1} \tilde{u}_{2} \tilde{u}_{3}\right)^{2}-\left(\tilde{u}_{1} \tilde{u}_{2} \tilde{u}_{3}\right)^{2} \\
& +\left(\tilde{u}_{1} \tilde{u}_{2} \tilde{u}_{3}\right)^{2}+\left(\tilde{u}_{1} \tilde{u}_{2} \tilde{u}_{3}\right)^{2}-\left(\tilde{u}_{1} \tilde{u}_{2} \tilde{u}_{3}\right)^{2} \\
& =0
\end{aligned}
$$

Therefore, the characteristic equation of $\boldsymbol{\sigma}$ in Eq.23 has three roots as follows

$$
\lambda_{1}=0, \lambda_{2}=I_{1}, \lambda_{3}=0 .
$$

From spectra representation theory of a symmetric tensor, we can rewrite the tensor $\boldsymbol{\sigma}$ as follows

$$
\begin{aligned}
\boldsymbol{\sigma} & =\lambda_{1} \hat{e}_{1} \otimes \hat{e}_{1}+\lambda_{2} \hat{e}_{2} \otimes \hat{e}_{2}+\lambda_{3} \hat{e}_{3} \otimes \hat{e}_{3} \\
& =\lambda_{2} \hat{e}_{2} \otimes \hat{e}_{2},
\end{aligned}
$$

where the base $\hat{\boldsymbol{e}}_{i}$ is an eigenvector of $\boldsymbol{\sigma}$, which can be determined by eigenvalue equation

$$
\boldsymbol{\sigma} \cdot \hat{e}=2 k \hat{e} \cdot \hat{e}
$$

where the fluctuation kinetic energy $k=\frac{1}{2}\left[\left(\tilde{u}_{1}\right)^{2}+\left(\tilde{u}_{2}\right)^{2}+\right.$ $\left.\left(\tilde{u}_{3}\right)^{2}\right]$, we find

$$
\hat{e}_{2}=\frac{1}{\sqrt{2 k}} \tilde{u}_{\ell} \boldsymbol{e}_{\ell}
$$

Hence we have

$$
\boldsymbol{\sigma}=\left[\left(\tilde{u}_{1}\right)^{2}+\left(\tilde{u}_{2}\right)^{2}+\left(\tilde{u}_{3}\right)^{2}\right] \hat{e}_{2} \otimes \hat{e}_{2},
$$

Therefore, the Reynolds tress tensor can be also given by

$$
\begin{aligned}
\boldsymbol{\tau} & =-\rho \lim _{T \rightarrow \infty} \frac{1}{T} \int_{t_{0}}^{t_{0}+T} \boldsymbol{\sigma} d t \\
& =-\rho \lim _{T \rightarrow \infty} \frac{1}{T} \int_{t_{0}}^{t_{0}+T}\left[\left(\tilde{u}_{1}\right)^{2}+\left(\tilde{u}_{2}\right)^{2}+\left(\tilde{u}_{3}\right)^{2}\right] \hat{e}_{2} \otimes \hat{e}_{2} d t
\end{aligned}
$$

It is proved once again that both the tensor $\boldsymbol{\sigma}$ and the Reynolds stress tensor $\boldsymbol{\tau}$ are the only function of $\tilde{u}_{1}, \tilde{u}_{2}, \tilde{u}_{3}$. As a summary, the number of unknowns of the Reynolds stress tensor is listed in the Table II:

TABLE I: Numbers of unknowns in the Reynolds stress

\begin{tabular}{c|c|c}
\hline & Current literatures & This paper \\
\hline Numbers & 6 & 3 \\
\hline Unknowns & $\tau_{11}, \tau_{12}, \tau_{13}, \tau_{22}, \tau_{23}, \tau_{33}$ & $\tilde{u}_{1}, \tilde{u}_{2}, \tilde{u}_{3}$ \\
\hline
\end{tabular}

Although the Reynolds-averaged Navier-Stokes (RANS) equations are unclosed, however, the four-equations RANS in Eqs.3 and 4 contain only 7 unknowns instead of 10 ones. The list of unknowns in the RANS are summarized in the below Table II:

TABLE II: Numbers of unknowns in the RANS

\begin{tabular}{c|c|c}
\hline & Current literature & This paper \\
\hline Numbers & 10 & 7 \\
\hline Unknowns & $\bar{u}_{1}, \bar{u}_{2}, \bar{u}_{3}$ & $\bar{u}_{1}, \bar{u}_{2}, \bar{u}_{3}$ \\
& $\bar{p}$ & $\bar{p}$ \\
& $\tau_{11}, \tau_{12}, \tau_{13}, \tau_{22}, \tau_{23}, \tau_{33}$ & $\tilde{u}_{1}, \tilde{u}_{2}, \tilde{u}_{3}$ \\
\hline
\end{tabular}

In the future, turbulence modelling will be focus on velocity fluctuations $\tilde{u}_{1}, \tilde{u}_{2}, \tilde{u}_{3}$ instead of the Reynolds stress $\tau_{i j}$. Advantage of modelling the velocity fluctuations $\tilde{u}_{1}, \tilde{u}_{2}, \tilde{u}_{3}$ is obvious, since it reduce the 6 components of $\tau_{i j}$ into 3 components of velocity fluctuations, and from experimental point of view, the components of velocity fluctuations are easy to be measured than the Reynolds stress tensor.

\section{TRANSPORT EQUATION OF REYNOLDS STRESS TENSOR}

With the above new understanding on the number of unknowns. In the following, we will present complete new perception on the higher-order correction of the Reynolds stress tensor.

In order to get more information about the Reynolds stress tensor, Reynolds [1] derived velocity fluctuation equations, and reformulated in index-tensorial form by Chou [4]. Here we present the equations in a bold-face 
tensorial form as follows

$$
\begin{gathered}
\rho \tilde{\boldsymbol{u}}_{, t}+\rho \boldsymbol{\nabla} \cdot(\overline{\boldsymbol{u}} \otimes \tilde{\boldsymbol{u}}+\tilde{\boldsymbol{u}} \otimes \overline{\boldsymbol{u}}+\tilde{\boldsymbol{u}} \otimes \tilde{\boldsymbol{u}})+\nabla \tilde{p} \\
=\mu \nabla^{2} \tilde{\boldsymbol{u}}+\rho \lim _{T \rightarrow \infty} \frac{1}{T} \int_{t}^{t+T} \boldsymbol{\nabla} \cdot(\tilde{\boldsymbol{u}} \otimes \tilde{\boldsymbol{u}}) d t \\
\boldsymbol{\nabla} \cdot \tilde{\boldsymbol{u}}=0 .
\end{gathered}
$$

According to the author's investigations [38-40], Eqs.3, 3,3 , and 3 form a closed integral-differential equations system, in which there are 8 equations with 8 unknowns $\overline{\boldsymbol{u}}, \tilde{\boldsymbol{u}}, \bar{p}, \tilde{p}$.

After some tensor algebra we arrive at an important equation on the Reynolds stress tensor as follows

$$
\begin{array}{r}
\frac{\partial \boldsymbol{\tau}}{\partial t}+\overline{\boldsymbol{u}} \cdot \nabla \boldsymbol{\tau}=-\boldsymbol{\tau} \cdot \boldsymbol{\nabla} \overline{\boldsymbol{u}}-\boldsymbol{\nabla} \overline{\boldsymbol{u}} \cdot \boldsymbol{\tau} \\
+2 \mu \boldsymbol{I}: \overline{\nabla \tilde{\boldsymbol{u}} \otimes \boldsymbol{\nabla} \tilde{\boldsymbol{u}}}+\overline{\tilde{\boldsymbol{u}} \otimes(\nabla \tilde{p})}+\overline{(\nabla \tilde{p}) \otimes \tilde{\boldsymbol{u}}} \\
+\mu \nabla^{2} \boldsymbol{\tau}+\rho \boldsymbol{\nabla} \cdot \overline{(\tilde{\boldsymbol{u}} \otimes \tilde{\boldsymbol{u}} \otimes \tilde{\boldsymbol{u}})}
\end{array}
$$

In current literature, it is believe that Eq.36 has 31 unknowns. However, we have complete different opinion. We believe the Reynolds stress equation Eq. 36 has only 7 unknowns. Specifically, accoutring for all symmetries, we have the following listed in the Table III.

TABLE III: Numbers of unknowns in the Reynolds stress equation

\begin{tabular}{c|c|c}
\hline & Current literature & This paper \\
\hline Numbers & 31 & 7 \\
\hline Unknowns & $\overline{\boldsymbol{u}}(3)$ & $\bar{u}_{1}, \bar{u}_{2}, \bar{u}_{3}$ \\
& $\tilde{\boldsymbol{u}} \otimes \tilde{\boldsymbol{u}} \otimes \tilde{\boldsymbol{u}}(10)$ & $\tilde{p}$ \\
& $\boldsymbol{I}: \frac{\nabla \tilde{\boldsymbol{u}} \otimes \boldsymbol{\nabla} \tilde{\boldsymbol{u}}}{(6)}$ & $\tilde{u}_{1}, \tilde{u}_{2}, \tilde{u}_{3}$ \\
& $\overline{\boldsymbol{u} \otimes(\nabla \tilde{p})}(6)$ & \\
& $\boldsymbol{\tau}(6)$ & \\
\hline
\end{tabular}

The above statement can be easily proved. For instance,

$$
\begin{array}{r}
\tilde{\boldsymbol{u}} \otimes \tilde{\boldsymbol{u}} \otimes \tilde{\boldsymbol{u}}=\overline{\tilde{u}_{i} \tilde{u}_{j} \tilde{u}_{k}} \boldsymbol{e}_{i} \otimes \boldsymbol{e}_{j} \otimes \boldsymbol{e}_{k} \\
=\left[\lim _{T \rightarrow \infty} \frac{1}{T} \int_{t_{0}}^{t_{0}+T}\left(\tilde{u}_{i} \tilde{u}_{j} \tilde{u}_{k}\right) d t\right] \boldsymbol{e}_{i} \otimes \boldsymbol{e}_{j} \otimes \boldsymbol{e}_{k},
\end{array}
$$

and

$$
\begin{array}{r}
\boldsymbol{I}: \overline{\boldsymbol{\nabla} \tilde{\boldsymbol{u}} \otimes \boldsymbol{\nabla} \tilde{\boldsymbol{u}}}=\left(\boldsymbol{e}_{k} \otimes \boldsymbol{e}_{k}\right):(\overline{\nabla \tilde{\boldsymbol{u}} \otimes \boldsymbol{\nabla} \tilde{\boldsymbol{u}}}) \\
\left.\left.=\overline{\left(\boldsymbol{e}_{k} \otimes \boldsymbol{e}_{k}\right):(\boldsymbol{\nabla} \tilde{\boldsymbol{u}} \otimes \boldsymbol{\nabla} \tilde{\boldsymbol{u}}}\right)=\overline{\left(\boldsymbol{e}_{k} \cdot \boldsymbol{\nabla} \tilde{\boldsymbol{u}}\right) \otimes\left(\boldsymbol{e}_{k} \cdot \boldsymbol{\nabla} \tilde{\boldsymbol{u}}\right.}\right) \\
\left.\left.=\overline{\left(\boldsymbol{\nabla}_{k} \tilde{\boldsymbol{u}}\right) \otimes\left(\boldsymbol{\nabla}_{k} \tilde{\boldsymbol{u}}\right.}\right)=\overline{\left(\nabla_{k} \tilde{u}_{i} \boldsymbol{e}_{i}\right) \otimes\left(\boldsymbol{\nabla}_{k} \tilde{u}_{j} \boldsymbol{e}_{j}\right.}\right) \\
\left.=\overline{\left(\boldsymbol{\nabla}_{k} \tilde{u}_{i}\right)\left(\boldsymbol{\nabla}_{k} \tilde{u}_{j}\right.}\right) \boldsymbol{e}_{i} \otimes \boldsymbol{e}_{j}=\frac{\partial \tilde{u}_{i}}{\partial x_{k}} \frac{\partial \tilde{u}_{j}}{\partial x_{k}} \boldsymbol{e}_{i} \otimes \boldsymbol{e}_{j} \\
=\left[\lim _{T \rightarrow \infty} \frac{1}{T} \int_{t_{0}}^{t_{0}+T}\left(\frac{\partial \tilde{u}_{i}}{\partial x_{k}} \frac{\partial \tilde{u}_{j}}{\partial x_{k}}\right) d t\right] \boldsymbol{e}_{i} \otimes \boldsymbol{e}_{j} .
\end{array}
$$

It is clear that mean value of $\overline{\tilde{u}_{i} \tilde{u}_{j} \tilde{u}_{k}}$ and $\overline{\frac{\partial \tilde{u}_{i}}{\partial x_{k}} \frac{\partial \tilde{u}_{j}}{\partial x_{k}}}$ can be calculated by the velocity fluctuations $\tilde{u}_{1}, \tilde{u}_{2}, \tilde{u}_{3}$. It means that $\tilde{u}_{1}, \tilde{u}_{2}, \tilde{u}_{3}$ are unknowns. Similarly,

$$
\begin{array}{r}
\overline{\tilde{\boldsymbol{u}} \otimes(\nabla \tilde{p})}=\overline{\tilde{u}_{i} \frac{\partial \tilde{p}}{\partial x_{j}}} \boldsymbol{e}_{i} \otimes \boldsymbol{e}_{j} \\
=\left[\lim _{T \rightarrow \infty} \frac{1}{T} \int_{t_{0}}^{t_{0}+T}\left(\tilde{u}_{i} \frac{\partial \tilde{p}}{\partial x_{j}}\right) d t\right] \boldsymbol{e}_{i} \otimes \boldsymbol{e}_{j} .
\end{array}
$$

The mean value of $\overline{\tilde{u}_{i} \frac{\partial \tilde{p}}{\partial x_{j}}}$ can be calculated by $\tilde{p}$ and $\tilde{u}_{1}, \tilde{u}_{2}, \tilde{u}_{3}$.

In the same way, we can still do the fourth order and higher orders as in [4] and textbooks. However, there is

\begin{tabular}{|c|c|c|}
\hline & Current literature & This paper \\
\hline Numbers & 21 & 7 \\
\hline Unknowns & $\begin{array}{c}k(3) \\
\overline{\boldsymbol{u}}(3) \\
\boldsymbol{\tau}(6) \\
\frac{\boldsymbol{\nabla} \tilde{\boldsymbol{u}}: \nabla \tilde{\boldsymbol{u}}}{\tilde{\tilde{p}} \tilde{\boldsymbol{u}}(3)} \\
\frac{[(\tilde{\boldsymbol{u}} \cdot \tilde{\boldsymbol{u}}) \tilde{\boldsymbol{u}}]}{(3)}\end{array}$ & $\begin{array}{c}\tilde{u}_{1}, \tilde{u}_{2}, \tilde{u}_{3} \\
\bar{u}_{1}, \bar{u}_{2}, \bar{u}_{3} \\
\tilde{p}\end{array}$ \\
\hline
\end{tabular}
no more unknowns can be created by any order equation about the Reynolds stress tensor.

\section{TRANSPORT EQUATION OF TURBULENCE KINETIC ENERGY}

If we do contraction operation for index $i$ and $j$ in the Eq.36, we have following transport equation for the turbulence kinetic energy $k$

$$
\begin{aligned}
& \rho\left(\frac{\partial k}{\partial t}+\overline{\boldsymbol{u}} \cdot \nabla k\right)=\boldsymbol{\tau}: \nabla \overline{\boldsymbol{u}}-\mu \overline{\boldsymbol{\nabla} \tilde{\boldsymbol{u}}: \nabla \tilde{\boldsymbol{u}}} \\
& \quad+\mu \boldsymbol{\nabla}^{2} k-\nabla \cdot(\overline{\tilde{p} \tilde{\boldsymbol{u}}})-\frac{1}{2} \rho \boldsymbol{\nabla} \cdot \overline{[(\tilde{\boldsymbol{u}} \cdot \tilde{\boldsymbol{u}}) \tilde{\boldsymbol{u}}]}
\end{aligned}
$$

where the kinetic energy $k=-\frac{1}{2} \tau_{k k}=\frac{1}{2} \overline{\tilde{u}_{k} \tilde{u}_{k}}=\frac{1}{2} \overline{\tilde{\boldsymbol{u}} \cdot \tilde{\boldsymbol{u}}}$. The number of unknowns in the kinetic energy equation Eq.40 is listed in the Table IV.

TABLE IV: Numbers of unknowns in the kinetic energy equation

In order to support the above understanding about the number of unknowns, let's present some examples in the following. The aim of doing these exercise are only for demonstration purpose: to show general algorithm to find turbulence solutions. We will not going to give complete solutions here, so that we can stay on our key focus, namely, to determine the number of unknowns in the Reynolds-averaged Navier-Stokes equations.

\section{EXAMPLE 1: ONE DIMENSIONAL BERGER'S EQUATION}

One dimensional Navier-Stokes equation, namely, Bergers's equation

$$
u_{, t}+u u_{, x}=\nu u_{, x x}+S(x, t),
$$


where the source term $S(x, t)$ is given by

$$
\begin{aligned}
S(x, t) & =\cos (x+t)+\nu \sin (x+t) \\
& +B \cos (x+t) \tanh \left(A-\frac{B}{2 \nu} x\right) \\
& +\sin (x+t) \cos (x+t) \\
& -\frac{1}{2 \nu} B^{2} \sin (x+t)\left[1-\tanh ^{2}\left(A-\frac{1}{2 \nu} B x\right)\right],
\end{aligned}
$$

where the trigonometric tangent function is defined as $\tanh (x)=\frac{\sinh (x)}{\cosh (x)}=\frac{e^{x}-e^{-x}}{e^{x}+e^{-x}}$, and $A, B$ are constant.

Substituting the Reynolds velocity decomposition $u=$ $\bar{u}(x)+\tilde{u}(x, t)$ into Eq.41, after time-averaging, we can get its RANS as follows

$$
\bar{u} \bar{u}_{, x}+\overline{\tilde{u} \tilde{u}_{, x}}=\nu \bar{u}_{, x x}+\bar{S},
$$

namely

$$
\bar{u} \bar{u}_{, x}+\lim _{T \rightarrow \infty} \frac{1}{T} \int_{t_{0}}^{t_{0}+T} \tilde{u} \tilde{u}_{, x} d t=\nu \bar{u}_{, x x}+\bar{S},
$$

where the mean source term $\bar{S}=\frac{1}{2}$. Obviously, the Eq.44 has only two unknowns, one is the mean velocity $\bar{u}$ and another is velocity fluctuation $\tilde{u}$, as stated in the previous part.

Since we have only one integral-differential equation Eq.44, therefore, the Eq.44 is not closed. To solve the Eq.44, we have to model either the mean field $\bar{u}$ or fluctuation $\tilde{u}$.

We can use the steady solution of $\bar{u} \bar{u}_{, x}=\nu \bar{u}_{, x x}$ as the 1 st order approximate solution of mean field. Hence, the 1st order exact solution is given by

$$
\bar{u}(x)=B_{1} \tanh \left(A_{1}-\frac{B_{1}}{2 \nu} x\right),
$$

where the constants $A_{1}, B_{1}$ will be determined later.

For fluctuation field $\tilde{u}$, since its mean $\tilde{u}$ must be zero, namely, $\lim _{T \rightarrow \infty} \frac{1}{T} \int_{t_{0}}^{t_{0}+T} \tilde{u} d t \equiv 0$, hence, we can take the 1 st order velocity fluctuation proposed by Reynolds [1] as an approximate solation

$$
\tilde{u}(x, t)=\sqrt{2} a \sin (\omega t+\kappa x),
$$

where $a$, frequency $\omega$ and wave number $\kappa$ are to be determined.

Substituting both Eqs.45 and 46 into Eq.44, we obtain

$$
a=\frac{1}{\sqrt{2 \kappa}}
$$

Therefore, we have the 1st order velocity field as follows

$$
u(x, t)=B_{1} \tanh \left(A_{1}-\frac{B_{1}}{2 \nu} x\right)+\frac{1}{\sqrt{2 \kappa}} \sin (\omega t+\kappa x) .
$$

For initial-boundary conditions $u(0,0)=1, u_{, x}(0,0)=1$, we get $B_{1}=\sqrt{1+\nu}, \tanh \left(A_{1}\right)=\frac{1}{\sqrt{1+\nu}} u_{, t}(0,0)=$
$1, u_{, x x}(0,0)=1$, and obtain $\omega=\sqrt{\kappa}$ and $\kappa=$ $\frac{1+\nu+\sqrt{1+\nu}}{2 \nu^{2}}-1$.

Hence, the 1st order solution is obtained

$$
\begin{array}{r}
u(x, t)=\sqrt{1+\nu} \tanh \left(A_{1}-\frac{\sqrt{1+\nu}}{2 \nu} x\right) \\
+\frac{1}{\sqrt{2 \kappa}} \sin (\sqrt{\kappa} t+\kappa x) .
\end{array}
$$

(1) The 1 st order turbulence velocity $u_{1 s t}$ is different from the exact solution of the Bergers equation in Eq.41. The exact solution of Berger's equation is given by

$$
u_{\text {exact }}(x, t)=B \tanh \left(A-\frac{B}{2 \nu} x\right)+\sin (x+t) .
$$

(2) In particular, when $x \rightarrow \infty$, their asymptotic behavior are totally different. The exact solution is going to be $u_{\text {exact }}=B+\sin (x+t)$, due to the property of $\lim _{x \rightarrow \infty} \tanh (x) \rightarrow 1$, while the turbulence solution

$$
u(x, t)=\sqrt{1+\nu}+\frac{1}{\sqrt{2 \kappa}} \sin (\sqrt{\kappa} t+\kappa x) . .
$$

(3) The turbulence kinetic energy is given by $k=$ $1 /(2 \kappa)$

For a better approximation, the velocity fluctuations can be assumed as $\tilde{u}(x, t)=\sqrt{2} a(x) \sin (\omega t)$.

\section{EXAMPLE 2: TURBULENT BOUNDARY LAYER FLOW OF SEMI-INFINITE SURFACE}

Here velocity components $u=u_{1}, v=u_{2}$, directions $1 \rightarrow x, 2 \rightarrow y$ are adopted respectively.

According to Prandtl's boundary layer concept [2, 42], the two dimensional Navier-Stokes equations can be simplified to following boundary layer equations

$$
\begin{aligned}
u_{, t}+u u_{, x}+v u_{, y} & =U \frac{d U}{d x}+\mu u_{, y y}, \\
u_{, x}+v_{, y} & =0
\end{aligned}
$$

where $U(x)$ is incoming velocity, $U=$ const. for infinite surface, hence $U_{, x}=0$.

In duct and boundary layer flow, we can approximate with excellent accuracy a simpler turbulent boundary layer equations

$$
\begin{aligned}
\rho\left(\bar{u} \bar{u}_{, x}+\bar{v} \bar{u}_{, y}\right) & =U \frac{d U}{d x}+\tau_{x x, x}+\tau_{x y, y}, \\
\bar{u}_{, x}+\bar{v}_{, y} & =0
\end{aligned}
$$

where total stress

$$
\begin{aligned}
& \tau_{x x}=-\rho \overline{\tilde{u} \tilde{u}}=-\rho \lim _{T \rightarrow \infty} \frac{1}{T} \int_{t_{0}}^{t_{0}+T} \tilde{u} \tilde{u} d t \\
& \tau_{x y}=\mu \bar{u}_{, y}-\rho \overline{\tilde{u} \tilde{v}}=\mu \bar{u}_{, y}-\rho \lim _{T \rightarrow \infty} \frac{1}{T} \int_{t_{0}}^{t_{0}+T} \tilde{u} \tilde{v} d t .
\end{aligned}
$$


To solve the above equations, we firstly omit the fluctuation term and reduce Eqs.54 and 55 into followings

$$
\begin{aligned}
\rho\left(\bar{u} \bar{u}_{, x}+\bar{v} \bar{u}_{, y}\right) & =\mu \bar{u}_{, y y}, \\
\bar{u}_{, x}+\bar{v}_{, y} & =0
\end{aligned}
$$

Blasius [42] has obtained their solutions as follows

$$
\begin{aligned}
& \bar{u}=U f^{\prime}(\xi), \\
& \bar{v}=\frac{1}{2} \sqrt{\frac{\nu U}{x}}\left(\xi f^{\prime}-f\right),
\end{aligned}
$$

where $\xi=y \sqrt{\frac{U}{\nu x}}, U$ is incoming velocity, $f^{\prime}=\frac{d f}{d \xi}$, and function $f(\xi)$ is the solution of equation $f f^{\prime \prime}+2 f^{\prime \prime \prime}=0$ under conditions $f(0)=f^{\prime}(0)=0, f^{\prime}(\infty)=1$.

For fluctuation field $\tilde{u}$ and $\tilde{v}$, since their mean must be vanish, hence, we can take the 1st order velocity fluctuation suggested by Reynolds [1] as their solutions

$$
\begin{aligned}
& \tilde{u}=\sqrt{2} c_{1} \cos (\varphi), \\
& \tilde{v}=\sqrt{2} c_{2} \cos (\varphi),
\end{aligned}
$$

where phase function $\varphi=\omega t+\kappa_{x} x+\kappa_{y} y$, and constants $c_{1}, c_{2}$.

Substituting Eqs. 60,61,62 and 63 into Eq. 54, we obtain

$$
\left(c_{1}^{2}\right) \kappa_{x}+\left(c_{1} c_{2}\right) \kappa_{y}=U \frac{d U}{d x}=0
$$

to determine $c_{1}, c_{2}$, we need mass conversation law of fluctuation field $\tilde{u}_{, x}+\tilde{v}_{, y}=0$, namely $\kappa_{x}=-\kappa_{y}=\kappa$, find the $c_{1}=c_{2}=C$, hence, we can write down velocity field as follows

$$
\begin{aligned}
& u=U f^{\prime}(\xi)+\sqrt{2} C \cos (\omega t+\kappa x-\kappa y), \\
& v=\frac{1}{2} \sqrt{\frac{\nu U}{x}}\left(\xi f^{\prime}-f\right)+\sqrt{2} C \cos (\omega t+\kappa x-\kappa y),
\end{aligned}
$$

where frequency $\omega$, wave number $\kappa$ and constant $\mathrm{C}$ can be determined by 3 initial-boundary conditions.

In the future, for a better approximation, we can take $\tilde{u}=\sqrt{2} c_{1}(x, y) \cos (\omega t)$ and $\tilde{v}=\sqrt{2} c_{2}(x, y) \cos (\omega t)$.

We must point out here that the one and/or two dimensional Navier-Stokes model can not represent a real turbulence, since turbulence motion is always three dimensional [7].

\section{EXAMPLE 3: THREE DIMENSIONAL TURBULENT FLOW SOLUTION ALGORISM}

Although there are different views about turbulence, there is a consensus that the deterministic Navier-Stokes equation probably contains all information, which is relevant to turbulence [18].
For three dimensional turbulent flow, we propose following algorithm:

(1) Find solution of corresponding equations of steady flow $\overline{\boldsymbol{u}}$ :

$$
\nabla \cdot(\overline{\boldsymbol{u}} \otimes \overline{\boldsymbol{u}})=\nu \nabla^{2} \overline{\boldsymbol{u}}
$$

(2) Calculate pressure (source term) $\bar{p}$ :

$$
\nabla^{2} \bar{p}=-\rho \boldsymbol{\nabla} \cdot(\overline{\boldsymbol{u}} \cdot \boldsymbol{\nabla} \overline{\boldsymbol{u}})
$$

(3) Propose an approximate solution for velocity fluctuations $\tilde{\boldsymbol{u}}$ :

$$
\tilde{\boldsymbol{u}}=\sqrt{2} \boldsymbol{C}(\boldsymbol{x}) \cos (\omega t)
$$

(4) Calculate the Reynolds stress tensor $\boldsymbol{\tau}$ :

$$
\begin{aligned}
\boldsymbol{\tau} & =-\rho \lim _{T \rightarrow \infty} \frac{1}{T} \int_{t}^{t+T}(\tilde{\boldsymbol{u}} \otimes \tilde{\boldsymbol{u}}) d t \\
& =-\rho(\boldsymbol{C} \otimes \boldsymbol{C}) .
\end{aligned}
$$

(5) Determine the coefficient vector $\boldsymbol{a}_{n}$ : Substitute $\overline{\boldsymbol{u}}, \bar{p}, \boldsymbol{\tau}$ into the RANS, hence we have

$$
\boldsymbol{\nabla} \cdot(\overline{\boldsymbol{u}} \otimes \overline{\boldsymbol{u}})-\nu \nabla^{2} \overline{\boldsymbol{u}}=-\frac{1}{\rho} \boldsymbol{\nabla} \bar{p}-\boldsymbol{\nabla} \cdot(\boldsymbol{C} \otimes \boldsymbol{C}) .
$$

Since $\overline{\boldsymbol{u}}$ is the solution of $\boldsymbol{\nabla} \cdot(\overline{\boldsymbol{u}} \otimes \overline{\boldsymbol{u}})=\nu \nabla^{2} \overline{\boldsymbol{u}}$, therefore, the left side of the above equation is zero, hence

$$
\boldsymbol{\nabla} \cdot\left(\boldsymbol{C} \otimes \boldsymbol{C}+\frac{1}{\rho} \bar{p} \boldsymbol{I}\right)=0,
$$

This relation reveals that the fluctuation magnitude is proportional to pressure, the turbulence can not maintained without continua source supplying.

(6) Calculate pressure fluctuation $\tilde{p}$

$$
\nabla^{2} \tilde{p}=-\rho \nabla \cdot[\overline{\boldsymbol{u}} \cdot \nabla \tilde{\boldsymbol{u}}+\tilde{\boldsymbol{u}} \cdot \nabla \overline{\boldsymbol{u}}+\tilde{\boldsymbol{u}} \cdot \nabla \tilde{\boldsymbol{u}}]-\boldsymbol{\tau} .
$$

(7) Calculate total pressure $p$ :

$$
p=\bar{p}+\tilde{p} .
$$

(8) Calculate total velocity field $\boldsymbol{u}$

$$
\boldsymbol{u}=\overline{\boldsymbol{u}}+\boldsymbol{C}(x) \cos \omega t .
$$

In the similar way, you can get higher order solutions.

\section{CONCLUSIONS}

In summary, this article has re-visited a fundamental problem in turbulence analysis, namely, how many unknowns are there in the Reynolds stress? This study not only clarified the number of unknowns in the formulations of the Reynolds-averaged Navier-Stokes equation, 
but also discovered the number of unknowns is much less than the conventional accoutring.

As application of this study, we have resolved the Berger's equation and turbulent Pranddtl boundary layer equations. All solutions have no any adjustable parameters. It is found that the Reynolds stress is mainly depends on the source term (ie. flow pressure), which means that the turbulence can not maintained if there is no source supplying.

Although the Reynolds-Averaged Navier-StokesEquations (RANS) has been formulated for more 100 years, unfortunately, all current literature have a wrong understanding on the numbers of unknowns. This situation must be corrected immediately, otherwise, it will be very harmful to scientific environment and definitely obstructing the understanding and solving of turbulence phenomena. The ideas and methodology of this paper are also applicable to the compressible turbulence Navier-Stokes equations. The study in the paper might shed a light to understand the well-known closure problem of turbulence, and will help to understand the puzzle and modelling of turbulence closure problem that has eluded scientists and mathematicians for centuries.

\section{Acknowledgements}

It is my great pleasure to have shared and discussed some of the above with Michael Sun from Bishops Diocesan College, whose pure and direct scientific sense inspired me.

[1] O. Reynolds, On the dynamical theory of incompressible viscous fluids and the determination of the criterion. Philos. Trans. R. Soc. 186:123 - 164(1895).

[2] L. Prandtl, On fluid motions with very small friction (in German). Third International Mathematical Congress, Heidelberg. 484-491(1904).

[3] L. Prandtl, Bericht uber die entstehung der turbulenz. Z. Angew. Math. Mech 5, 136(1925).

[4] P-Y. Chou, On velocity correlations and the solutions of the equations of turbulent fluctuation. Q. Appl. Math. 111(1):38-54(1945).

[5] P-Y. Chou and R.L. Chou, 50 years of turbulence research in China. Annu. Rev. Fluid Mech. 27:1-15(1995).

[6] P. Bradshaw, An Introduction to Turbulence and Its Measurement. Pergamon Press, New York (1971).

[7] H. Tennekes and J.L. Lumley, A First Course in Turbulence. Cambridge: The MIT Press (1972).

[8] D.C. Lesilie, Developments in the Theory of Turbulence. Clarendon Press, Oxford (1973).

[9] A.A. Townsend, The Structure of Turbulent Shear Flow 2nd ed., Cambridge University Press, New York (1976).

[10] S.Y. Chen and P.-Y. Zhou, The application of quasisimilarity conditions in turbulence modeling theory. Journal of Hydrodynamics, 2(2) (1987)
[11] M. Lesieur, Turbulence in Fluids. 2nd ed. Kluwer, Dordrecht (1990).

[12] D.C. Wilcox, Turbulence Modeling for CFD. D C W Industries (1993).

[13] S.B. Pope, Turbulent Flows. Cambridge University Press, Cambridge (2000).

[14] P.A. Davidson, Turbulence. Oxford University Press, Oxford (2004).

[15] B. Hof, Experimental Observation of Nonlinear Traveling Waves in Turbulent Pipe Flow. Science 305, 1594 (2004).

[16] G. Falkovich and K.R. Sreenivasan, Lessons from hydrodynamic turbulence. Physics Today, 43-49 (April 2006).

[17] C.B. Lee and J.Z. Wu, Transition in wall-bounded flows. Applied Mechanics Reviews, 61(3), 030802 (2008)

[18] U. Frisch, Turbulence: The Legacy of A.N. Kolmogorov. Cambridge University Press, Cambridge (2008).

[19] I. Marusic, R. Mathis and N. Hutchins, Predictive model for wall-bounded turbulent Flow. Science 329, 193 (2010).

[20] A.J. Smits, B.J. McKeon and I. Marusic, High-Reynolds Number Wall Turbulence, Annu. Rev. Fluid Mech. 43, 353 (2011).

[21] P.A. Davison, et al. A Voyage Through Turbulence. Cambridge: Cambridge University Press (2011).

[22] B. Suri, J.R. Tithof, R.O. Grigoriev and M.F. Schatz, Forecasting Fluid Flows Using the Geometry of Turbulence. Phys. Rev. Lett. 118, 114501 (2017).

[23] D. Castelvecchi, On the trial of turbulence. Nature, 548:382 (2017).

[24] L.D. Landau and E. M. Lifshitz, Mechanics (3rd ed.) (Butterworth-Heinemann, Oxford, 1976).

[25] A.N. Kolmogorov, The local structure of turbulence in incompressible viscous fluid for very large Reynolds number. Dokl. Akad. Nauk SSSR, 30:299-303 (1941a) (reprinted in Proc.R.Soc.Lond. A, 434,9-13, 1991).

[26] A.N. Kolmogorov, On degeneration (decay) of isotropic turbulence in an incompressible visous liquid. Dokl. Akad. Nauk SSSR, 31:538-540 (1941b).

[27] A.N. Kolmogorov, Dissipation of energy in locally isotropic turbulence. Dokl.Akad. Nauk SSSR, 32:1618 (1941c).(reprinted in Proc.R.Soc.Lond. A, 434,15-17, 1991).

[28] K.R. Sreenivasan, On the scaling of the turbulence energy dissipation rate. Physics of Fluids, 27,5:1048-1051 (1984).

[29] Z.S. She and E. Lévêque, Universal scaling laws in fully developed turbulence. Phys. Rev. Lett. 72,336(1994) .

[30] E.N. Lorenz, Deterministic non-periodic flow. J. Atmos Sci. 20:130 - 41(1963).

[31] R. Benzi, P. Paladin, G. Parisis and A. Vulpiani, On the multifractal nature of fully developed turbulence and chaotic systems. J. Phys. A: Math. Gen. 17:35213531(1984).

[32] S.A. Orszag and G.S. Patterson, Numerical simulation of three-dimensional homogeneous isotropic turbulence. Phys. Rev. Lett. 28,76(1972).

[33] B. Sun, The temporal scaling laws of compressible turbulence. Modern Physics Letters B. 30,(23) 1650297 (2016).

[34] B. Sun, Scaling laws of compressible turbulence. Appl. Math. Mech.-Engl. Ed. 38: 765(2017).

[35] B. Sun, Thirty years of turbulence study in China. Applied Mathematics and Mechanics (English Edition), 40(2), 193 - 214 (2019); https://doi.org/10.1007/s10483019-2427-9. 
[36] B. Sun, A additive decomposition of velocity gradient, Physics of Fluids 31, 061702(2019); doi:10.1063/1.5100872.

[37] B. Sun, On the Reynolds-averaged Navier-Stokes equations, Preprints, doi:10.20944/preprints201907.0038.v1.

[38] B. Sun, On closure problem of incompressible turbulent flow. Preprints 2018, 2018070622 (doi: 10.20944/preprints201807.0622.v2).

[39] B. Sun, An Intrinsic Formulation of Incompressible Navier-Stokes Turbulent Flow. Preprints 2018,
2018080071 (doi: 10.20944/preprints201808.0071.v3).

[40] Sun, B. A Novel Simplification of the ReynoldsChou-Navier-Stokes Turbulence Equations of Incompressible Flow. Preprints 2018, 2018070030 (doi: 10.20944/preprints201807.0030.v1).

[41] https://en.wikipedia.org/wiki/Dyadic_tensor

[42] H. Blasius, Grenzschichten in Fljssigkeiten mit kleiner Reibung. Z. Math.Phys., 56 1-37(1908). 